\title{
Spectral Deconvolution for Overlapping GC/MS Components
}

\author{
Bruce N. Colby \\ Pacific Analytical, Inc., Carlsbad, California, USA
}

\begin{abstract}
The use of mass chromatogram peak centroids has been investigated as a means of deconvoluting the spectra of overlapping gas chromatography/mass spectrometry components. The peak centroids have been calculated with a precision of 0.04 scans (sd). This proved sufficient to allow deconvolution of the mass spectra belonging to two chemical components which were eluted 0.48 scans apart. For a more complex chromatography peak, it was possible to deconvolute the spectra of six components which were eluted within a 9 scan window. All the spectra produced by using this deconvolution mechanism agreed well with National Institute of Standards and Technology database spectra. (J Am Soc Mass Spectrom 1992, 3, 558-562)
\end{abstract}

$\mathrm{W}$ Then samples are analyzed by gas chromatography/mass spectrometry (GC/MS), it is common to encounter situations where two or more components are eluted with retention times sufficiently close that the resulting total in current trace causes them to appear as if they were a single component. This situation is encountered frequently with samples derived from wastewater, hazardous waste, and plant and animal tissue. When spectra from these multicomponent peaks are interpreted manually, the process ranges from complex to nearly impossible. Library search programs provide some help but they are generally no better than the data submitted to them.

Most commercial GC/MS data systems provide some relief for situations where overlapping components are encountered by providing a routine based on the Biller-Biemann [1] algorithm. Here a spectrum is generated which incorporates mass/intensity pairs only from those mass to charge ratios which have mass chromatogram maxima at or adjacent to the selected scan. Thus, if two components have no common mass to charge ratios and they are separated by two or more scans, distinct spectra can be generated for each component. Although this algorithm is simple to implement, it frequently provides insufficient resolution to be useful.

A more powerful deconvolution algorithm based on what Dromey et al. [2] describe as "a relatively complex mathematical treatment of the GC peak profile" was reported capable of separating the spectra of components eluted as closely as 1.5 scans. The key concept in this algorithm was that all mass chro-

Address reprint requests to Bruce N. Colby, Pacific Analytical, Inc., 6349 Paseo Del Lago, Carlsbad, CA 92009. matogram peaks associated with a single component will have the same peak shape. The algorithm has not been implemented in a commercially available GC/MS data system, presumably due to its complexity.

Recently a study was undertaken in our laboratory to develop an improved mass spectral deconvolution algorithm. The goal was to extend the basic Biller-Biemann maximization concept to allow assessment of peak shape yet maintain sufficient simplicity to allow practical implementation in a commercial GC/MS data system. The purpose of this article is to describe a mass chromatogram centroid based deconvolution algorithm which has proven simple to implement and which produces useful mass spectra even when components are very closely eluted.

\section{Experimental}

The data presented here were collected on two different quadrupole GC/MS instruments. One was a VG 12-250 operated according to the specifications described in EPA Method 624 [3]. In summary, this involves a 2-m packed column, 3 second scans (cycle time) from $m / z 35$ to $m / z 250$, and sample introduction via purge and trap. Data from this instrument were acquired into a VG 11-250 PDP-11 based data system and the files were reformatted and transferred to a VG Lab-Base PC based data system for processing. Data were acquired for a $50 \mathrm{ng}$ Method 624 calibration solution obtained from Supelco.

The second instrument was a VG Trio- 1 operated according to the specifications in EPA Method 1625 [4]. This is an isotope dilution method which utilizes a 30-m fused silica capillary column, 1 second scans (cycle time) from $m / z 35$ to $m / z \quad 450$ and sample introduction via syringe injection. The Trio-1 data 
were acquired directly into the Lab-Base data system. Data were acquired for a $50 \mathrm{ng} / \mathrm{uL}$ decafluorotriphenylphosphine (DFTPP) solution and a Method 1625 initial precision and recovery solution. The naturally abundant compounds were obtained from Supelco, and the labeled analogs from Cambridge Isotopes. All library searching was performed using the "Iden" module provided with the Lab-Base data system and the most recently available National Institute of Standards and Technology database.

The deconvolution process consisted of calculating time centroids for each mass chromatogram maximum in the displayed data range. Because the data were stored in mass-intensity format, it was necessary to account for the time between scan start and the time a mass was actually measured. If the data had been stored in time-intensity format, this would not have been necessary.

For simplicity only three data points were used for the centroid calculation, the maximum plus the points on each side. The centroid was defined as the time at which the first derivative of the quadratic equation which exactly fit the three points was equal to zero. The intensity of the mass chromatogram maximum was corrected for any baseline signal, assigned a retention time based on centroid location, and the results placed in a mass/intensity/retention time array. Once all the mass chromatogram maxima had been processed, the array was sorted on retention time. A second array was then constructed with ten elements per scan. Intensities from the first array were summed into the second array such that intensities with the same retention times were added to the intensity sum in the same array element. This produced what could be considered a deconvoluted total ion current (DTIC) with a resolution of 0.1 scans. When all the data had been processed, the intensity sums in the DTIC array were plotted as intensity versus retention time. An example of such a trace is given in Figure 1.

By working backwards from the DTIC trace, a mass spectrum was generated for an intensity maximum by locating its start and end points in terms of retention time. These time limits were then used to select which mass/intensity pairs in the first array were to be associated with the mass spectrum of the deconvoluted TIC maximum.

\section{Results and Discussion}

For the approach described above to be useful, mass chromatogram centroids for a single component must be tightly grouped. This was verified by investigating the TIC peak containing DFTPP (Figure 1a). DFTPP was chosen because it contains mass peaks across a wide range [5] and hence provides a worst case situation for centroid precision evaluation. For these data the mass chromatogram peak centroids for all masses with intensities greater than or equal to $1 \%$ of the base peak had a standard deviation of 0.04 scans. The effect of this precision is clearly illustrated by the narrow peak in the DTIC trace (Figure 1b). It is worth noting that there is a significant difference between the retention time labels of the TIC (12.315 minutes) and DTIC (12.368 minutes) peaks tops shown in Figure 1 . This is because the data system sets the retention time of a scan to the time the scan was started. The DTIC peak top, on the other hand, is based on the times at which the mass chromatograms maximized. Although it is possible for TIC and DTIC times to coincide, it is more likely that the mass chromatogram maxima will have maxima with times that are greater than the time associated with scan start time.

Based on the mass chromatogram centroid precision noted above, it should be possible to identify which mass peaks belong to components which differ in retention time by as little as a quarter of a scan with about a $99 \%$ confidence. To determine if such resolution was possible, a TIC peak (Figure 2a) containing hexadecane- $\mathrm{d}_{34}$ was investigated for the presence of

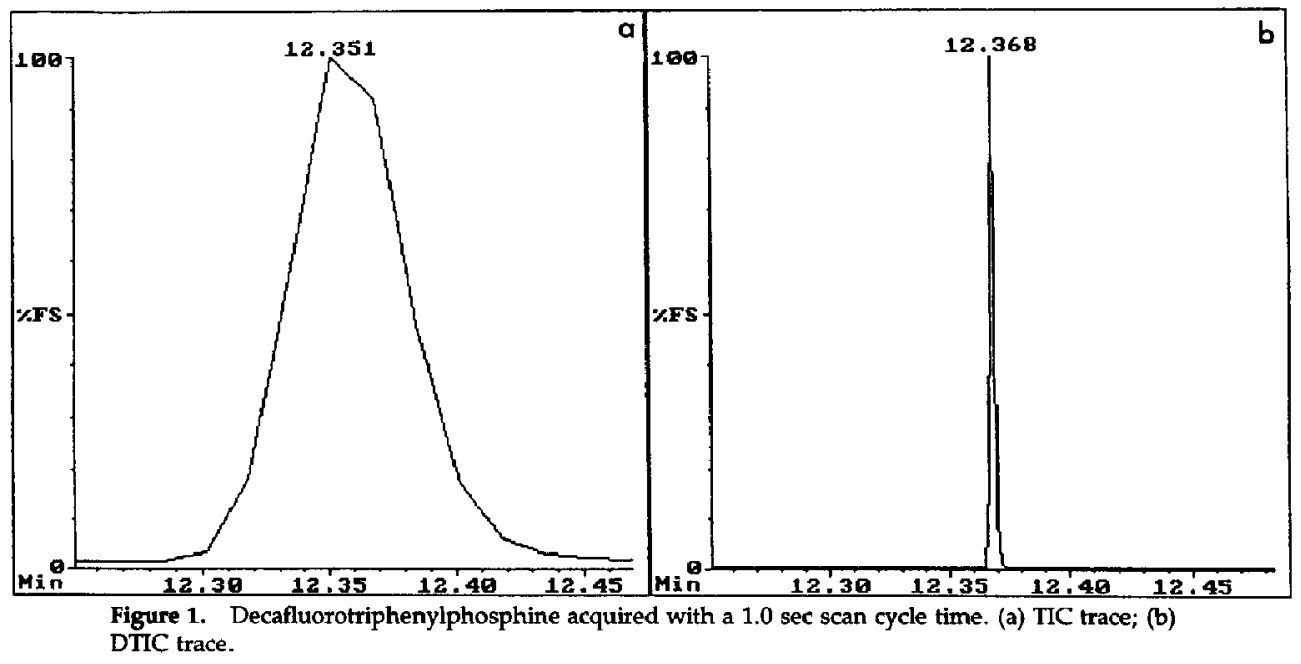




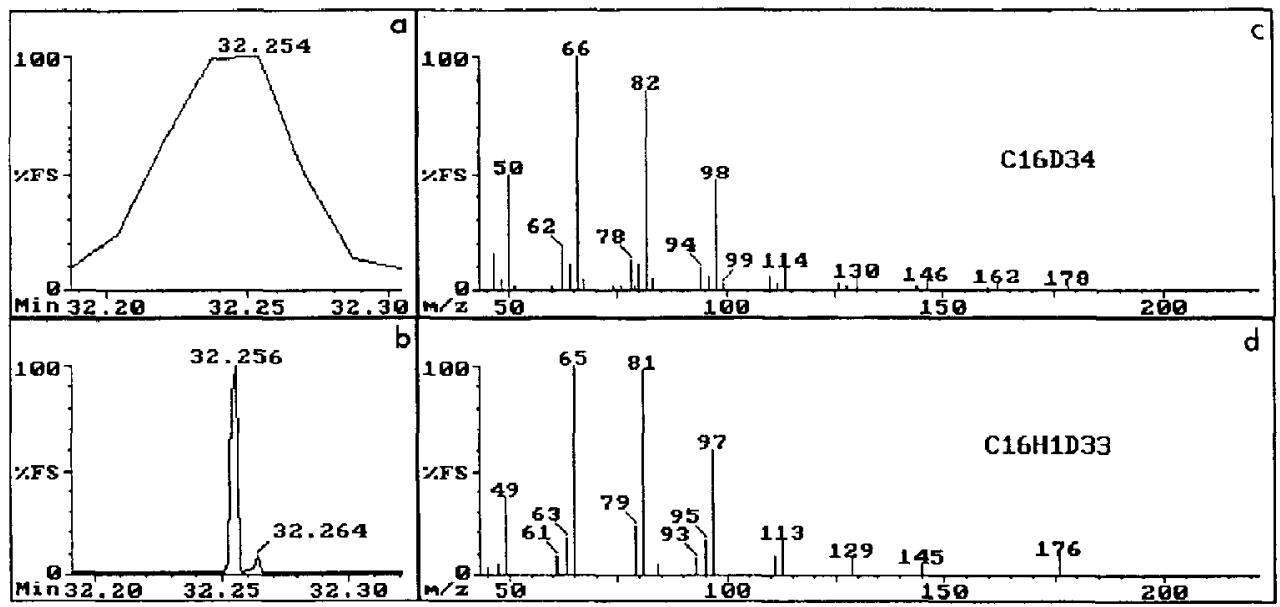

Figure 2. Hexadecane- $d_{34}$. (a) TIC trace; (b) DTIC trace; (c) deconvoluted mass spectrum of hexadecane- $\mathrm{d}_{34} ;$ (d) deconvoluted mass spectrum of hexadecane- $\mathrm{d}_{33}$. These data were acquired with a 1.0 second scan cycle time.

any hexadecane- $d_{33}$ impurity. The DTIC trace generated (Figure $2 b$ ) clearly indicated the presence of a second peak with a slightly longer retention time than the main peak. On review of the spectra associated with the two centroid groupings, the first produced a good spectra for hexadecane- $\mathrm{d}_{34}$ at 32.256 minutes (Figure $2 \mathrm{c}$ ), and the second a very reasonable spectrum for hexadecane- $d_{33}$ at 32.264 minutes (Figure $2 \mathrm{~d}$ ). The 0.008 minute difference in retention time between the two peaks represents 0.48 scans.

Two aspects of the hexadecane- $\mathrm{d}_{33}$ spectrum are worth noting. First, the spectrum does not exhibit the expected peaks for $m / z 50,66,82$, etc., which would be expected due to the roughly $3 \%$ chance that an ion fragment would not contain the hydrogen atom. This is because the hexadecane- $d_{33}$ represents only about $8 \%$ of the TIC peak and intensity of these peaks would be very low compared to those of the hexadecane- $\mathrm{d}_{34}$. Consequently, the intensity from these peaks is included in the hexadecane- $d_{34}$ spectrum.

The second notable aspect of the hexadecane- $\mathrm{d}_{33}$ spectrum is the peak at $m / z 176$, which logic dictates should be at $m / z 177$. Detailed review of the raw data indicates that this peak most likely belongs to the hexadecane- $\mathrm{d}_{34}$ spectrum but that, due to poor ion statistics with such a low level signal, it just happens to result in a centroid which is aligned with the hexadecane- $\mathrm{d}_{33}$ centroids.

Based on the above hexadecane data, it is clear that mass chromatogram centroid deconvolution can only generate useful spectra when there is a sufficient difference in retention time between the overlapped components and when the components have spectra with dissimilar mass peaks. Also, the ability to deconvolute spectra by using this algorithm will be dependent on having high quality ion statistics. If the acquired data have a poor signal-to-noise ratio, related mass chromatograms will not form a tight group of centroids.
The deconvolution algorithm was also tested on several multicomponent peaks in the other two solutions analyzed. Two examples of these are described below. The first was a three component peak in the Method 624 calibration standard. The TIC trace is shown in Figure 3a. Visual inspection of this peak provides no indication of multiple components. Carrying out a library search on the spectrum obtained at the peak maximum resulted in a misleading answer no matter how the search parameters were set. Performing a Biller-Biemann type deconvolution using the Lab-Base Refine command did not change the data spectrum significantly. The minor changes it induced resulted in different, but not improved, search results.

When this TIC peak is subjected to mass chromatogram centroid analysis, three clear groupings are observed in the DTIC trace (Figure 3b). The closest pair was separated by 0.017 minutes; the other pair by 0.046 minutes. These separations correspond to 0.34 and 0.92 scans, respectively. The spectra generated from these groupings were library searched and each produced the correct answer as the best hit (Figure $4 a-c)$.

The second example of overlapped components is present in the Method 1625 solution where there were six components in a single TIC peak which exhibited a slight shoulder on one side (Figure 5a). As was true in the Method 624 example above, when the spectrum associated with the TIC peak maximum was searched, no useful results were generated. Applying the Biller-Biemann algorithm had some positive effect in that some mass peaks associated with the shoulder were removed. However, there were still so many components present that no real improvement in the search result was realized.

When this TIC peak was subjected to mass chromatogram centroid analysis, six clear groupings were observed in the DTIC trace (Figure 5b). The closest 


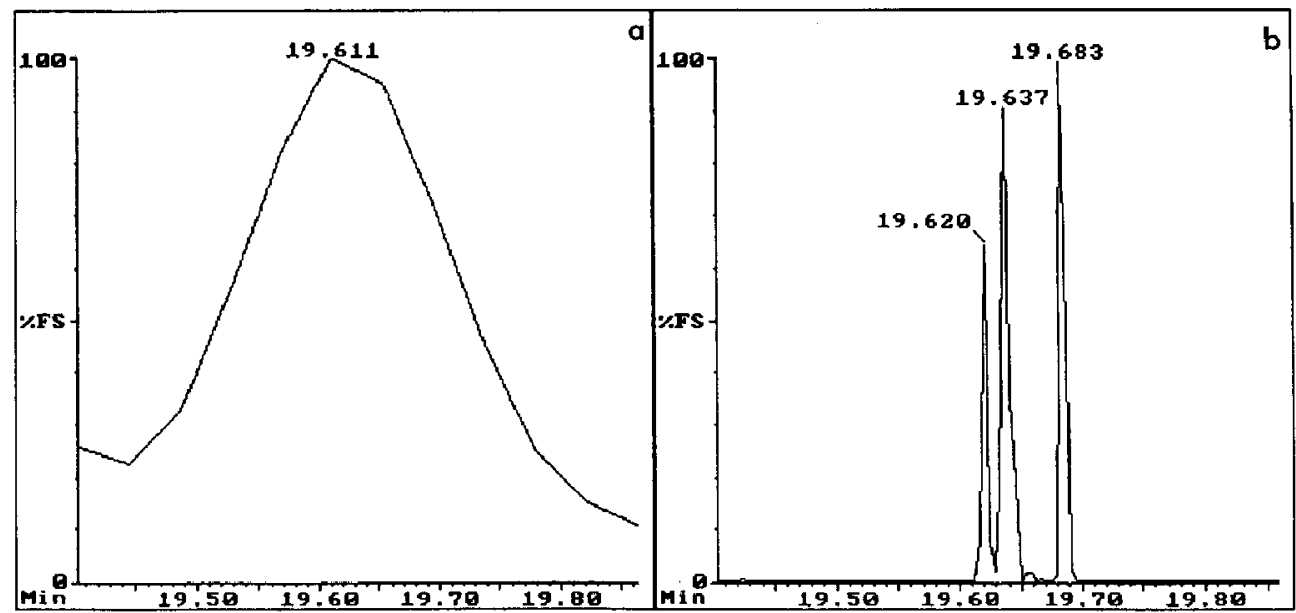

Figure 3. Three component peak. (a) TIC trace; (b) DTIC trace. These data were acquired with a 3.0 second scan cycle time.

pair was separated by 0.015 minutes or 0.90 scans; the next closest pair by 0.021 minutes or 1.26 scans. The spectra generated from the closest peaks in this grouping were searched. They all produced the correct answer as the best hit with the exception of the saturated hydrocarbon, which does not have a distinctive spectrum (Figure 6a-c).

The remaining three peaks in this group were biphenyl- $d_{10}(18.750 \mathrm{~min})$ and 2-chloronaphthalene- $d_{7}$ $(18.788 \mathrm{~min})$, which were not represented in the library, and 2,3,6-trichlorophenol (18.883 $\mathrm{min})$. It is interesting to note that the two intensity array peaks associated with the deuterated compounds have partially resolved shoulders with slightly longer retention times than the main peaks. Inspecting the spectra of these small shoulder peaks indicated that their masses were associated with $d_{9}$ and $d_{6}$ impurities, respectively.

\section{Conclusions}

Using a three-point quadratic fit to determine mass chromatogram peak centroids and then establishing groupings of related masses based on retention time is an excellent way to deconvolute the mass spectra of overlapped components. The data presented demonstrate this for spectra submitted to library search but the improvement would also be available for spectra intended for manual spectral interpretation.

One as yet uninvestigated aspect of this form of deconvolution which applies equally as well to single component TIC peaks is the possibility of assigning

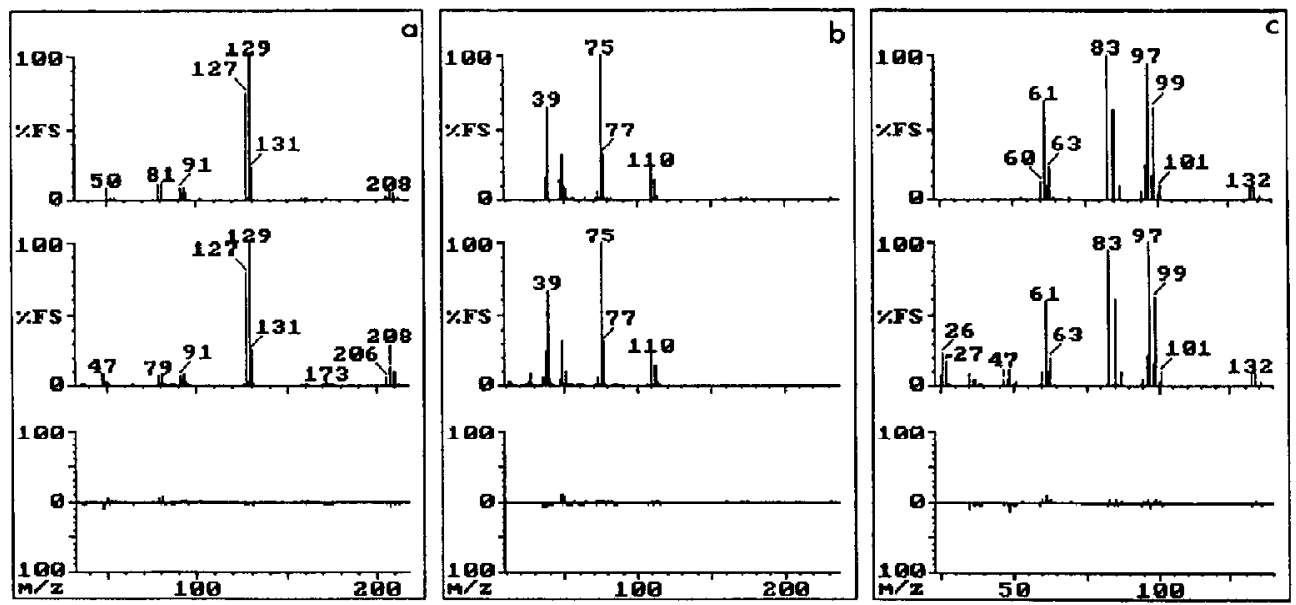

Figure 4. Library search results for deconvoluted spectra in the three component peak. (a) Dibromochloromethane; (b) 1,3-dichloro-1-propene; (c) 1,1,2-trichloroethane. The top trace shows the deconvoluted mass spectrum; the center trace the best fit National Institute of Standards and Technology library spectrum; the bottom trace the difference between the two above traces. 


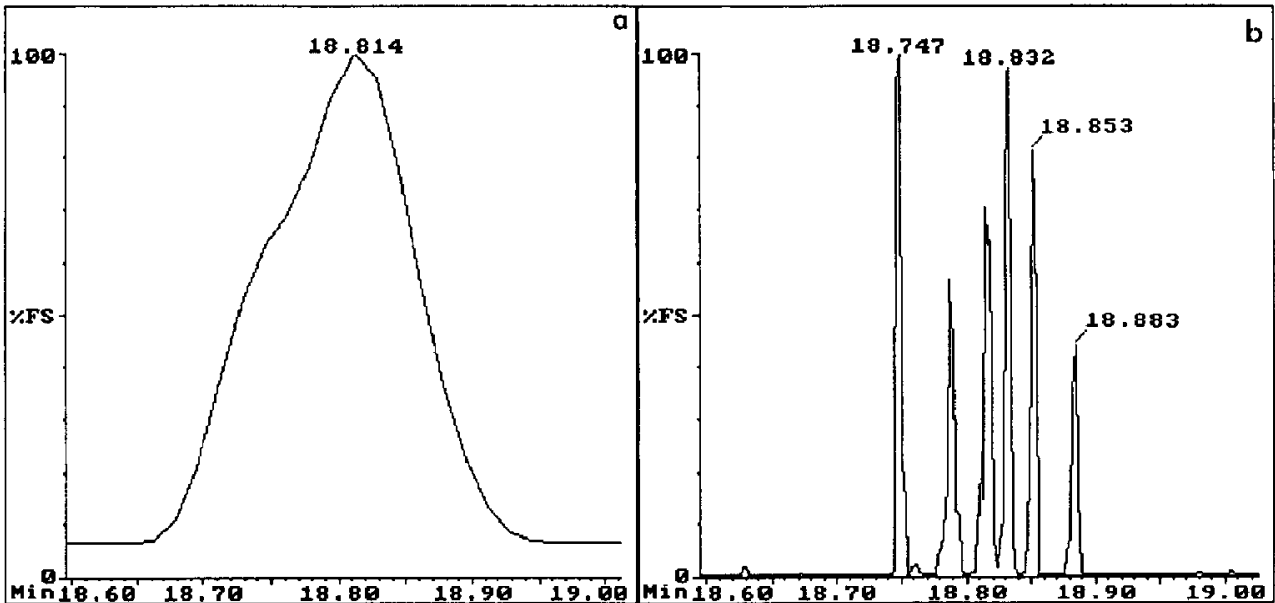

Figure 5. Six component peak. (a) TIC trace; (b) DTIC trace. These data were acquired with a 1,0 second scan cycle time.
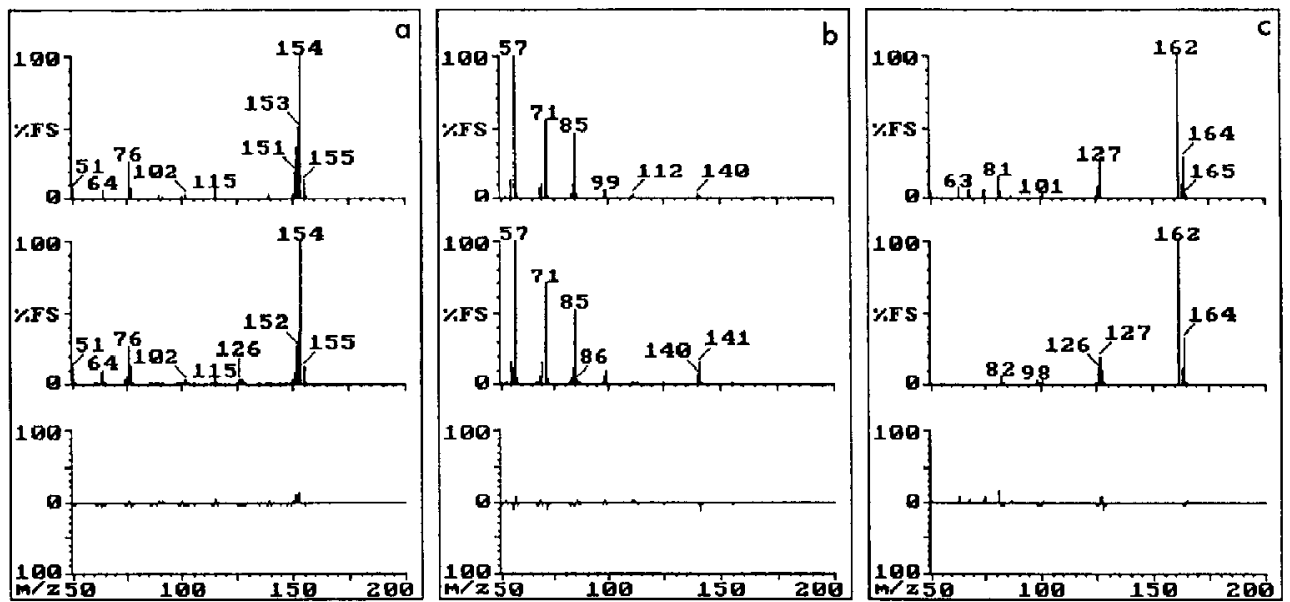

Figure 6. Library search results for deconvoluted spectra from the six component peak. (a) Third component, biphenyl; (b) fourth component, n-tetradecane; (c) fifth component, 2-chloronaphthalene. The top trace shows the deconvoluted mass spectrum; the center trace the best fit National Institute of Standards and Technology library spectrum; the bottom trace the difference between the two above traces.

intensity based on the peak height of the mass chromatogram at its centroid. In principle this should provide a mechanism to eliminate the skewing of spectra associated with scanning the mass spectrometer during acquisition. This is currently being coded and its impact will be tested in the future.

\section{Acknowledgment}

The author thanks VG Masslab for its support and encouragement, and for incorporating the deconvolution algorithm into an experimental version of their Lab-Base software for testing and graphics generation.

\section{References}

1. Biller, J. E.; Biemann, K. Anal. Letters 1974, 7, 515.

2. Dromey, R. G.; Stefik, M. J.; Rindfleisch, T. C.; Duffielk, A. M. Anal. Chem 1976, 48, 1365.

3. Method 624, Federal Register, Vol. 49, No. 209, October 26, 1984, page 141 .

4. Method 1625, Federal Register, Vol. 49, No. 209, October 26, 1984 , page 184 .

5. Eichelberger, J. W.; Harris, L. E.; Budde, W. L. Anal. Chem. $1975,47,995$. 